\title{
Toxoplasma encephalitis due to highly active antiretroviral therapy non-compliance
}

\author{
Muhammad Ali Shahid, Fuad Bashjawish, Mohamed Elshazzly
}

\begin{abstract}
Introduction: The most common opportunistic infection and cause of CNS infection in untreated acquired immunodeficiency syndrome (AIDS) patients is Toxoplasma Encephalitis caused by the parasite Toxoplasma gondii. Toxoplasmosis usually causes infection in AIDS patients (defined as $\mathrm{CD}_{4}<200$ cells/microL) when CD4 counts drop below 100 cell/microL and reactivation when the $\mathrm{CD} 4$ counts fall below 200 cells $/ \mu l$. Whether a primary infection or reactivation, immunocompromised persons present with serious symptoms such as headache, confusion, seizures, and blurred vision which can indicate necrotizing encephalitis. Without treatment, patients progress to coma in days to weeks, significantly increasing morbidity and mortality. Case Report: We present a 52-year old African American female who presented with a two-day history of chest pain, productive cough and shortness of breath. She has a past medical history of recurrent Pneumocystis Jiroveci pneumonia, HIV, and a 4 year history of non-compliance with anti-retroviral therapy. Her last CD4 count, measured 132 cells $/ \mathrm{mm}^{3}$ four weeks prior to admission. Within 24
\end{abstract}

Muhammad Ali Shahid', Fuad Bashjawish², Mohamed Elshazzly ${ }^{1}$

Affiliations: ${ }^{1}$ Campbell University School of Osteopathic Medicine, Lillington, NC, USA; ${ }^{2}$ Department of Internal Medicine, Cape Fear Valley Hospital, Fayetteville, NC, USA.

Corresponding Author: Mohamed Elshazzly, Campbell University School of Osteopathic Medicine, 4350 US-421, Lillington, NC 27546; Email: Meelshazzly0201@email.campbell.edu

Received: 11 March 2018

Accepted: 02 April 2018

Published: 18 April 2018 hours of admission, the patient developed two seizures, became comatose, and was admitted to the intensive care unit. Imaging studies of the head and brain showed a $3.7 \times 3.9 \mathrm{~cm}$ solid cystic mass in the left parietotemporal lobe and an accompanying subarachnoid hemorrhage. The patient was started on TrimethoprimSulfamethoxazole (TMP-SMX) for a possible diagnosis of toxoplasmosis. The following day, the patient had multiple bradycardic episodes possibly due to spontaneous breathing trial attempts, but was still unresponsive. The family was consulted on the patient's status, and they decided that aggressive therapy be stopped for the patient. Unfortunately, the patient expired the following day. Conclusion: This case highlights the different treatments of toxoplasmosis in AIDS patient according to the standard of care, as well as overlapping CNS complications that can be fatal. Thus, highlighting the importance of early detection and prevention in the immunocompromised individuals.

Keywords: Acquired immunodeficiency syndrome, Encephalitis, Highly active antiretroviral therapy, Toxoplasmosis

\section{How to cite this article}

Shahid MA, Bashjawish F, Elshazzly M. Toxoplasma encephalitis due to highly active antiretroviral therapy non-compliance. Int $\mathrm{J}$ Case Rep Images 2018;9:100907Z01MS2018.

Article ID: 100907Z01MS2018

$$
* * * * * * * * *
$$

doi: 10.5348/100907Zo1MS2018CR 


\section{EDORIUM Journals}

Int J Case Rep Images 2018;9:100907Z01MS2018.

www.ijcasereportsandimages.com

\section{INTRODUCTION}

Toxoplasmosis is the most common central nervous system (CNS) infection in patients with Human Immunodeficiency Virus (HIV) with a $\mathrm{CD}_{4}$ count $<200$ cells $/ \mathrm{mm}^{3}$ who are not receiving prophylactic treatment [1]. The disease manifests itself in the advancing stages of the HIV infection. As high as $30 \%$ of patients with AIDS and a CD4 count less than 100 cells $/ \mathrm{mm}^{3}$ with no prophylactic treatment present with reactivation of Toxoplasmosis [2]. Toxoplasmosis is acquired through the ingestion of infectious parasitic oocysts transmitted either through soil, contaminated cat feces, or undercooked meat. The most common presenting symptoms in a patient with CNS toxoplasmosis are headache and confusion, with other less specific symptoms such as fever, seizures, and focal neurological deficits. The best way to prevent a patient from acquiring CNS toxoplasmosis is by restoring cellular immunity with antiretroviral therapy, avoiding contact with cat feces or eating undercooked meat. We report the case of a woman who was non-compliant with highly active antiretroviral therapy (HAART) therapy and a decreasing CD4 count who expired following imaging and serology results consistent with Toxoplasma encephalopathy.

\section{CASE REPORT}

The patient was a 52-year-old African American female who presented to the hospital with a 2-day history of chest pain, productive cough and shortness of breath. The patient was admitted to the hospital three months prior for Pneumocystis Jiroveci pneumonia that was effectively treated with Trimethoprim-Sulfamethoxazole (TMP-SMX). The patient was discharged to a long term acute care, and effectively finished her treatment of antibiotics two days prior to new admission. The patient had a past medical history of HIV and non-compliance with HAART therapy. Her last CD4 count was measured four weeks prior to admission, and it was 132 cells $/ \mathrm{mm}^{3}$.

Vitals signs showed hypertension and tachycardia, and our patient was on $100 \%$ oxygen on a $2 \mathrm{~L}$ nasal cannula. Additional laboratory reports revealed an elevated White blood cell (WBC) count of $17.2 \times 10^{3} / \mathrm{uL}$, a decreased Red Blood Cell (RBC) count of $3.43 \times 10^{6} / \mathrm{uL}$, a decreased Hemoglobin of $9.9 \mathrm{~g} / \mathrm{dL}$, and a decreased Hematocrit of $31.3 \%$. The patient also had an increased Lactate Dehydrogenase (LDH) of 306 units/L and was slightly hyponatremic. Her HIV1-RNA PCR levels were 180 copies/ml (Table 1 ).

Upon admission, the patient was cachectic, short of breath and producing green to yellow sputum. Sputum cultures were obtained, which grew Pseudomonas aeruginosa. Based on susceptibility testing, the patient was given IV Amikacin, Colistin, and Vancomycin in the Emergency Department. Our patient then developed progressive respiratory symptoms with reported chest pains and elevated troponins (1.500 ng/ml). Electrocardiogram (EKG) findings were unremarkable for any ischemic changes. She was given therapeutic Lovenox for pulmonary embolism (PE) prophylaxis. CT-angiogram (CTA) was negative for any PE but showed multifocal pneumonia with cavitation in the superior segment of the lower lobe. Within the next 24 hours, the patient had 2 seizures, became comatose, and was admitted to the ICU. The patient was placed on a ventilator, and a Computed Tomogram (CT) of the head and brain were ordered. According to the neurosurgeon, the CT scan showed a $3.7 \times 3.9 \mathrm{~cm}$ solid cystic mass with blood fluid levels in the cystic area in the left parietotemporal lobe (Figure 1). The patient was started on IV Levetiracetam for her seizure activity and placed on an increased dose of TMP-SMX for a possible diagnosis of Toxoplasmosis. Additionally, HIVPCR and Toxoplasma IgG titers were ordered (Table 1).

The following morning, the patient had an acute bleed from her left internal jugular vein, and was given one unit of packed RBC, which increased her hemoglobin from 6.4 $\mathrm{g} / \mathrm{dl}$ to $7.9 \mathrm{~g} / \mathrm{dl}$. The patient was still comatose, and her

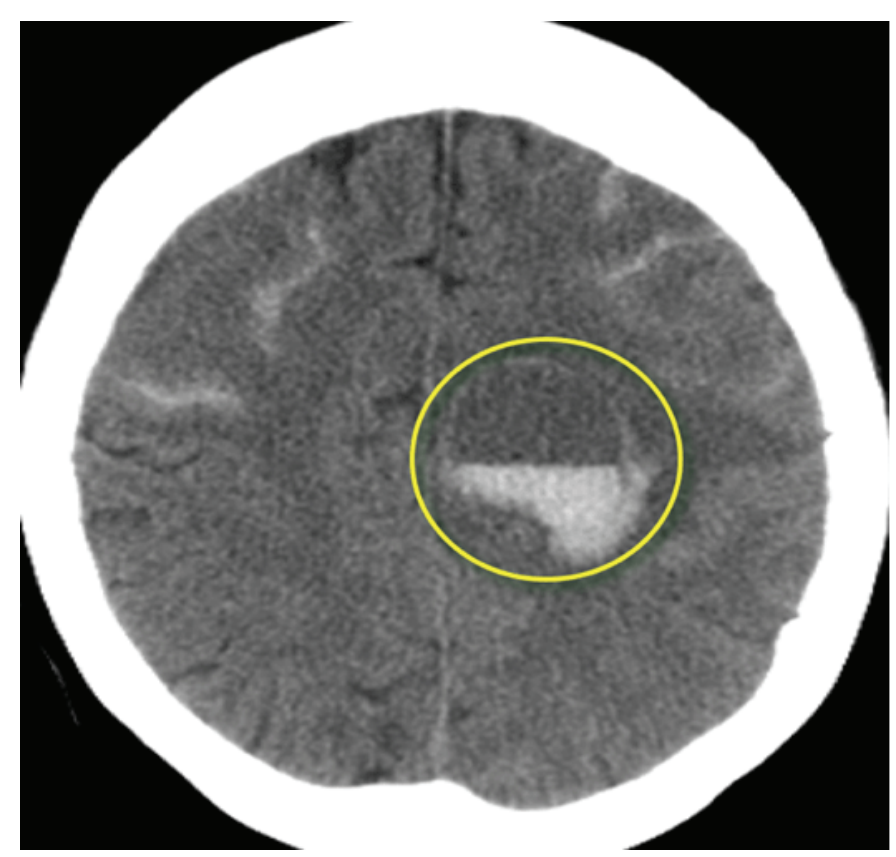

Figure 1: A 3.7x3.9 cm solid cystic mass with blood fluid levels in the cystic area in the Fronto-temporal lobe on an axial CT image through the head without contrast.

Table 1: Blood monitoring during the first five days of hospitalization.

\begin{tabular}{lccccc} 
& Day & Day & Day & Day & Day \\
& $\mathbf{1}$ & $\mathbf{2}$ & $\mathbf{3}$ & $\mathbf{4}$ & $\mathbf{5}$ \\
WBC & 17.2 & 24.2 & 27.7 & 23.6 & 16.0 \\
Procalcitonin & 0.08 & - & 0.80 & - & 1.01 \\
HIV-1 RNA PCR & \multicolumn{5}{c}{180} \\
RPR & \multicolumn{5}{c}{ Non-Reactive } \\
Toxoplasma IgG & \multicolumn{5}{c}{65.1} \\
Log-10 HIV-1 RNA & \multicolumn{5}{c}{2.255} \\
\hline
\end{tabular}




\section{EDORIUM Journals}

Procalcitonin levels increased to $0.8 \mathrm{ng} / \mathrm{ml}$ from $0.08 \mathrm{ng} /$ ml. Her Toxoplasmosis Ig-G came back positive, with a level of $65.1 \mathrm{IU} / \mathrm{ml}$. She was continued on an increased dose of TMP-SMX for her seropositive Toxoplasma, as well as her current medication regimen.

The following day, the patient had multiple bradycardic episodes possibly due to spontaneous breathing trial attempts. The patient was still comatose and had $6 \mathrm{~mm}$ unresponsive pupils. The family was consulted on the patient's status, and they decided that aggressive therapy be stopped for the patient. The patient expired the next day.

\section{DISCUSSION}

Our patient's detrimental clinical course underscores the importance of medical compliance with HAART therapy for HIV patients. Carefully examining the actions taken place along the hospital timeline shows that most medical actions taken were warranted and appropriate given the clinical context at that time. This patient had been complaining of shortness of breath, chest pain, and had an elevation in her troponins without corresponding ECG changes. These symptoms, in the absence of any focal neurological complaints or symptoms of headache or seizures, weighed in favor of anticoagulation with Lovenox to treat ischemic cardiac demand or possible PE. It was only after the intended therapeutic Lovenox was initiated that an intracranial bleed had formed, and the misstep was realized by the team. Although the team thought that an infection was her primary cause of symptoms, her presentation of chest pain, shortness of breath without being febrile led the team to believe that a PE was likely.Our patient was immunocompromised and in a hypercoagulable state due to underlying infection and inflammation, and the dose of Lovenox was given concurrently with the CTA. Obtaining a D-Dimer wouldn't have been useful in her situation, as she was already in a state of infection, as it would have been increased.It is thought that our patient had a possible mycotic aneurysm rupture due to bacterial colonization. The cause was unclear, but we postulate that it could be due to her multi-drug resistant Pseudomonas pneumonia, which she tested positive for in her blood cultures. It was her intracranial bleed that caused her to have two seizures, by which time the patient had already become neurologically unresponsive. Although reversal of Lovenox with Protamine Sulfate was attempted, it would not change her clinical course as she remained neurologically unresponsive.

A review of the current literature shows how to avoid similar outcomes in future patients, especially those who are non-compliant with their antiretroviral medications. According to a study by Jones et al., HIV-associated toxoplasmosis hospitalizations dropped markedly after 1995 when highly active antiretroviral therapy was introduced [3]. According to another study by Basavaraju et al., it is recommended that all HIV-infected individuals be tested for baseline IgG antibodies to Toxoplasma to detect any latent infection [4]. In addition, all HIVinfected individuals should be counseled regarding the exposure to Toxoplasma infections, such as avoiding eating raw or undercooked meat and avoiding exposure to feline excrement or litter boxes. While CT scan is the first imaging modality to detect CNS lesions, MRI is more sensitive at detecting the presence of tell-tale ring-enhancing lesions [2]. This can help differentiate Toxoplasma encephalitis from primary CNS lymphoma. In addition, newer imaging techniques, such as signal photon emission CT or positron emission tomography can enhance specificity to rule out other CNS lesions such as lymphomas.

Initial treatment with Sulfadiazine and Pyrimethamine, as well as alternative treatments with Trimethoprim-Sulfamethoxazole, have proven effective in controlling the disease process [5]. Oral Clindamycin with Pyrimethamine have also proven to be effective in the treatment of Toxoplasma Encephalitis (TE). In one study by Luft et al., 71\% of patients responded to six weeks of treatment with oral clindamycin and pyrimethamine [6]. In another study by Ammassari et al., the frequency of TE decreased from 72.2 to 18.6 percent with TMPSMX prophylaxis [7]. Primary prophylactic treatment of TE efficacy comes from retrospective analyses of trials evaluating antimicrobial treatment to prevent Pneumocystis infections [7]. Prophylactic treatment of toxoplasmosis with TMP-SMX is recommended when a patient is asymptomatic, has a CD4 count less than 100 cells $/ \mathrm{mm}^{3}$ with positive T. gondii IgG serology, or has a CD4 count less than 200 cells $/ \mathrm{mm}^{3}$ and has an opportunistic infection developing [8]. Patients who deteriorate neurologically or don't improve despite 1014 days of appropriate treatment should be considered candidates for brain biopsy [6].

HAART therapy adherence has been shown to be the most effective way in preventing opportunistic infections in AIDS patients. Since our patient was non-compliant with HAART therapy, she was at an increased risk for these infections, including Toxoplasmosis. In a cohort collaboration study by Trickley et al., between 1996 and 2010, life expectancy in 20-year-old patients starting HAART increased by about 9 years in women and 10 years in men [9]. If our patient had been more compliant with her HAART therapy, she could have possibly avoided these serious complications. In another study by Silva et al., which compared an autopsy review of CNS lesions in AIDS patients pre- and post-HAART therapy in Brazil, Toxoplasmosis was less common in the HAART group (17.8\%) vs the non-HAART group (36.2) [10]. HAART therapy has been shown to be the most beneficial for patients to prevent these often-fatal complications, and we postulated that if she had been more adherent with her therapy, her prognosis would have been more promising.

One final salient feature we would like to discuss is the fact that our patient's mental status was gradually 


\section{EDORIUM Journals}

declining; during the weeks before she had her two seizures in the hospital. She was evaluated by a psychiatrist and was determined to be medically incompetent to make her own decisions due to AIDS encephalopathy versus dementia.

Our patient likely had a combination of a TE and formation of a solid cystic mass that bled into the brain parenchyma. Before such a hazardous clinical course evolves, it is highly recommended to perform serological testing for likely infectious causes, such as Toxoplasmosis, Aspergillosis, Syphilis, Tuberculosis, and Nocardia to monitor a patient's infective status. Should an HIV/AIDS patient be non-compliant with his/her HAART therapy, we recommend having a family member monitor his/ her oral intake of the medication or having close followup with an infectious disease or primary care physician. Doing so will ensure that they have CD4 counts above 200 cells $/ \mathrm{mm}^{8}$, which can offer some protection to the multiple aforementioned opportunistic organisms.

\section{CONCLUSION}

The aforementioned article notes that some patients with TE can also present with neuropsychiatric disorders including psychosis, dementia, anxiety, and personality disorders. Building an appropriate differential by having an infectious cause in mind can prompt earlier serological testing, which can help avoid the formation of insidious CNS diseases with appropriate prophylactic treatment.

\section{REFERENCES}

1. Porter SB, Sande MA. Toxoplasmosis of the central nervous system in the acquired immunodeficiency syndrome. N Engl J Med 1992 Dec 3;327(23):1643-8.

2. Grant IH, Gold JW, Rosenblum M, Niedzwiecki D, Armstrong D. Toxoplasma gondii serology in HIV-infected patients: The development of central nervous system toxoplasmosis in AIDS. AIDS 1990 Jun;4(6):519-21.

3. Jones JL, Roberts JM. Toxoplasmosis hospitalizations in the United States, 2008, and trends, 1993-2008. Clin Infect Dis 2012 Apr;54(7):e58-61.

4. Basavaraju A. Toxoplasmosis in HIV infection: An overview. Trop Parasitol 2016 Jul-Dec;6(2):129-35.

5. Connolly MP, Haitsma G, Hernández AV, Vidal JE. Systematic review and meta-analysis of secondary prophylaxis for prevention of HIV-related toxoplasmic encephalitis relapse using trimethoprimsulfamethoxazole. Pathog Glob Health 2017 Sep;111(6):327-31.

6. Luft BJ, Hafner R, Korzun AH, et al. Toxoplasmic encephalitis in patients with the acquired immunodeficiency syndrome. Members of the ACTG 077p/ANRS oog Study Team. N Engl J Med 1993 Sep 30;329(14):995-1000.

7. Ammassari A, Scoppettuolo G, Murri R, et al. Changing disease patterns in focal brain lesion- causing disorders in AIDS. J Acquir Immune Defic Syndr Hum Retrovirol 1998 Aug 1;18(4):365-71.

8. Schneider MM, Hoepelman AI, Eeftinck Schattenkerk $\mathrm{JK}$, et al. A controlled trial of aerosolized pentamidine or trimethoprim-sulfamethoxazole as primary prophylaxis against Pneumocystis carinii pneumonia in patients with human immunodeficiency virus infection. The Dutch AIDS treatment group. N Engl J Med 1992 Dec 24;327(26):1836-41.

9. Trickey A, May MT, Vehreschild JJ, et al. Survival of HIV-positive patients starting antiretroviral therapy between 1996 and 2013: A collaborative analysis of cohort studies. Lancet HIV 2017 Aug;4(8):e349-56.

10. Silva AC, Rodrigues BS, Micheletti AM, et al. Neuropathology of AIDS: An autopsy review of 284 cases from Brazil comparing the findings pre- and post-HAART (highly active antiretroviral therapy) and pre- and postmortem correlation. AIDS Res Treat 2012;2012:186850.

$* * * * * * * * *$

\section{Author Contributions}

Muhammad Ali Shahid - Substantial contributions to conception and design, Acquisition of data, Drafting the article, Revising it critically for important intellectual content, Final approval of the version to be published Fuad Bashjawish - Substantial contributions to conception and design, Drafting the article, Revising it critically for important intellectual content, Final approval of the version to be published

Mohamed Elshazzly - Substantial contributions to conception and design, Acquisition of data, Drafting the article, Revising it critically for important intellectual content, Final approval of the version to be published

\section{Guarantor of Submission}

The corresponding author is the guarantor of submission.

\section{Source of Support}

None

\section{Consent Statement}

Written informed consent was obtained from the patient for publication of this case report.

\section{Conflict of Interest}

The authors whose names are listed immediately below certify that they have NO affiliations with or involvement in any organization or entity with any financial interest (such as honoraria; educational grants; participation in speakers' bureaus; membership, employment, consultancies, stock ownership, or other equity interest; and expert testimony or patentlicensing arrangements), or non-financial interest (such as personal or professional relationships, affiliations, knowledge or beliefs) in the subject matter or materials discussed in this manuscript. 


\section{Copyright}

(C) 2018 Muhammad Ali Shahid et al. This article is distributed under the terms of Creative Commons Attribution License which permits unrestricted use, distribution and reproduction in any medium provided the original author(s) and original publisher are properly credited. Please see the copyright policy on the journal website for more information.
Access full text article on other devices

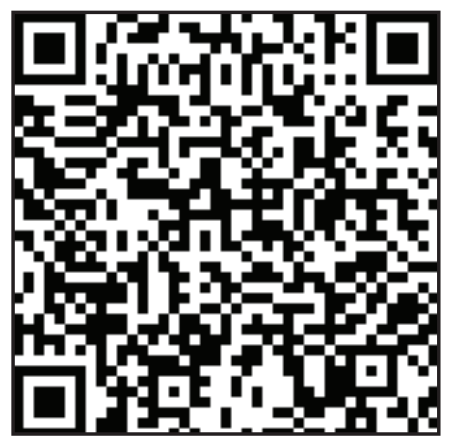

Access PDF of article on other devices

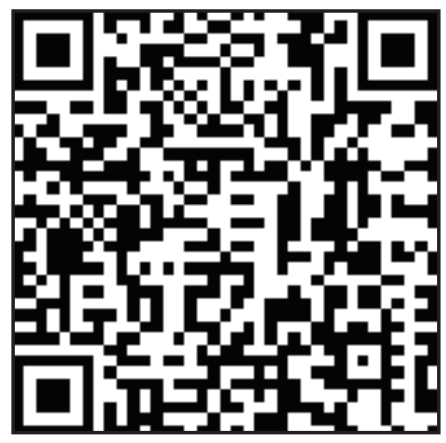

